\title{
Estado del arte sobre problemáticas financieras de las pymes en Bogotá, Colombia y América Latina
}

\section{State of the art on financial problems of SMEs in Bogotá, Colombia and Latin America}

DOI: http://dx.doi.org/10.17981/econcuc.39.2.2018.05

Artículo de revisión. Fecha de recepción: 27/03/2018 Fecha de aceptación: 23/08/2018

\author{
Ana María Gamez Tellez \\ Universidad de La Salle, Bogotá, D.C. (Colombia) \\ agamez28@unisalle.edu.co \\ Mabel Cristina Morales Bautista \\ Universidad de La Salle, Bogotá, D.C. (Colombia) \\ mmorales09@unisalle.edu.co \\ Cindy Tatiana Ramirez López \\ Universidad de La Salle, Bogotá, D.C. (Colombia) \\ cramirez48@unisalle.edu.co
}

Para citar este artículo:

Gamez, A., Morales, M. y Ramirez, C. (2018). Estado del arte sobre problemáticas financieras de las pymes en Bogotá, Colombia y América Latina. Económicas CUC, 39(2). 77-94. DOI: http://dx.doi.org/10.17981/econcuc.39.2.2018.05

\section{Resumen}

Gran parte de las empresas latinoamericanas son clasificadas como pequeñas y medianas empresas (Pymes) y actualmente enfrentan algunos obstáculos frente a su crecimiento y permanencia en el mercado; debido a un inadecuado manejo financiero que genera problemáticas en las demás áreas de estas compañías. El abordaje metodológico del presente estudio asume un enfoque cualitativo con alcance descriptivo de tipo documental. A partir de la revisión sistemática, se evidenció la relevancia que tienen los factores asociados de innovación y tecnología, acceso a crédito, productividad y competitividad, planificación y asignación de recursos, configuración de la estructura de capital, manejo financiero y políticas públicas. Estos factores, afectan principalmente los objetivos financieros, la liquidez, rentabilidad y solvencia, lo cual causa problemáticas financieras y la destrucción de valor en las Pymes.

Palabras clave: Pymes, objetivos financieros, liquidez, rentabilidad, solvencia.

\section{Abstract}

Most Latin American companies are classified as small and medium enterprises (SMEs) and currently face some obstacles to their growth and permanence in the market; due to inadequate financial management that generates problems in the other areas of these companies. The methodological approach of the present study assumes a qualitative approach with a descriptive scope of documentary type. From the systematic review, the relevance of the associated factors of innovation and technology, access to credit, productivity and competitiveness, planning and allocation of resources, the configuration of the capital structure, financial management, and public policies was evidenced. These factors mainly affect financial objectives, liquidity, profitability, and solvency, which causes financial problems and the destruction of value in SMEs.

Keywords: SMEs, financial objectives, liquidity, profitability, solvency. 


\section{Introducción}

El presente artículo revisa y analiza el estado del arte que existe sobre los factores que originan problemáticas financieras de las pymes en Bogotá, Colombia y América Latina. Teniendo en cuenta la importancia que las Pymes tienen sobre la relevancia de la economía latinoamericana, desde un punto de vista social y económico, ya que contribuyen de manera significativa en la producción y el empleo de estos países.

Debido a lo anterior, es indispensable conocer los factores de este caso de estudio en el área financiera, que generan efectos negativos en el crecimiento, y la permanencia de estas empresas en el mercado; y a raíz de esto no es posible pensar en la internacionalización como respuesta a un mundo globalizado. Entre los factores que generan problemas se analizan: la productividad y competitividad, el acceso al crédito, la planificación y asignación de recursos, la configuración de la estructura de capital, las políticas públicas y el manejo financiero.

Esta revisión resulta pertinente, debido a que las pymes no tienen un índice alto de supervivencia en los países latinoamericanos y se debe en muchos casos a un mal manejo financiero. Por tal razón, conocer y determinar los principales factores que se han estudiado y evaluado por diferentes autores resulta relevante. Adicionalmente, los trabajos encontrados no presentan un informe completo con las problemáticas evidenciadas para estas empresas, sino que se enfocan en analizar un factor específico en una región determinada, por lo cual se consideran importante realizar una recopilación de las mismas.

Enfocar el análisis en Bogotá, Colombia y América Latina es importante, por- que según la Comisión Económica para América Latina y el Caribe (CEPAL, s.f.), aunque las pymes conforman el $99 \%$ de la industria y son las generadoras de la mayoría de los empleos, tienen una productividad muy baja en comparación con grandes empresas y podría estar relacionado con factores financieros.

En síntesis, esta investigación se inicia con una revisión de los factores generales que originan las problemáticas financieras, para luego determinar que aspectos afectan y en que manera se generan las problemáticas en las pequeñas y medianas empresas en Bogotá, Colombia y América Latina.

\section{Desarrollo y análisis}

Factores que originan las problemáticas financieras en las pymes

\section{- Innovación y tecnología}

Para algunos autores la tecnología y la innovación influyen en la manera como las empresas manejan sus recursos, toman decisiones y crean ventajas competitivas, que se ven reflejadas en diferentes ámbitos de la empresa. Por esta razón, se recopilan diferentes artículos que mencionan los factores que pueden generar ventajas y problemas en las Pymes.

En el caso de Gálvez, Riascos y Contreras (2014), en su escrito sobre la influencia de la tecnología en las mipymes, indican que el bajo uso de la tecnología por parte de las empresas en Colombia puede llegar a presentar una desventaja en nivel de competencia, lo que a la vez genera un impacto negativo sobre su rentabilidad. De igual manera, Villareal, Arias, Salas y Holguín (2014) se refieren a la innovación como influencia sobre la productividad de acuerdo con 
el tamaño de las empresas y en donde se encuentran, lo cual para las pymes, con una baja participación en el factor de investigación y desarrollo se traduce en bajos rendimientos para las mismas.

Lambardi y Mora (2014), siguiendo la corriente de los anteriores autores, resaltan como para los países en desarrollo la carencia de innovación, afecta negativamente la productividad de las empresas y la capacidad de toma de decisiones. Para Jara, Guerrero, Jiménez, Hernández y Rubio (2014), quienes investigaron las pymes del sector de restaurantes en la Candelaria-Bogotá, los elementos como el uso de la tecnología, la capacitación de los trabajadores e inseguridad, son factores que afectan la competitividad en estos negocios.

Adicionalmente, Cruz, Pérez y Piedra (2014), usando estadística descriptiva encontraron una correlación positiva entre crecimiento empresarial y competitividad e innovación, que se incrementaría si se tienen nuevos softwares en producción, maquinaria de tecnología, campañas de marketing y capacitaciones. Así mismo, Estrada, Cuevas y Cortes (2015) encuentran una correlación positiva de las variables de innovación y rendimiento, dando conclusiones en donde el problema al que se enfrentan muchas empresas de eficiencia e innovación se puede resolver realizando mejoras y cambios que permitan generar rentabilidad.

También para García-Pérez, GálvezAlbarracín y Maldonado-Guzmán (2016), en su estudio, con una muestra de 386 pymes de Latinoamérica, donde verifican a través de un modelo econométrico de regresiones lineales múltiples por mínimos cuadrados ordinarios (MCO), midiendo la correlación de las variables de innovación en productos, en procesos y en gestión. Dando como resultado el hecho de encontrar que sin innovación no existirá rendimiento empresarial y no se logra mejorar la competitividad de las empresas. En el caso de Córdoba (2015), al analizar pequeñas y medianas empresas de un sector de confecciones ubicado en la ciudad de Medellín, encuentra que la tecnología es un determinante en la productividad y competitividad de las empresas debido a que sus inversiones se dirigen a procesos productivos, dejando a un lado las inversiones tecnológicas, lo cual les permitiría llegar a diferentes mercados.

Para Valencia, Trochez, Vanegas y Restrepo (2016), quienes analizan el riesgo de quiebra en el sector agroindustrial antioqueño a través de un modelo matemático-financiero lineal mixto, donde encuentran problemáticas referentes a la falta de innovación, tecnológia y apoyo al pequeño productor, que pueden afectar al fortalecimiento de capacidades y las condiciones para la competitividad en el mercado externo. Del mismo modo, Franco y Urbano (2016) realizaron un estudio en el departamento del Valle de Cauca y confirman que los empresarios no prestan suficiente importancia a los aspectos tecnológicos, lo cual genera debilidades en las pymes por su capacidad de producción y diferenciación.

Por otro lado, Riascos, Aguilera y Achicanoy (2016), concluyen que una falencia para las pymes es una mayor inversión en hardware que en software, por lo cual no se agilizan procesos en la compañía dificultando la creación de valor en el manejo de datos. Para Carrillo, Franky, Páez y Pedraza (2016), la productividad está asociada a la implementación de herramientas tecnológicas como el procesamiento en línea de pedidos, por lo cual las Pymes pueden tener implicaciones y afectaciones en la agilidad de la empresa a las demandas del mercado. 
Autores como Bedoya, López y Marulanda (2016), concluyen que las tecnologías de la información tienen influencia sobre estas empresas, al permitir mejorar su desarrollo, aprovechar ventajas y obtener rendimientos mayores, y de esta manera la baja adopción de estas herramientas ocasiona deficiencias en estos factores. También, Zumba, Torres y Aguilar (2016) reafirman que el uso de la tecnología es de provecho para las empresas, pues el uso de páginas web y redes sociales les ofrece una gran ventaja frente a las empresas en el mercado que no implementan la tecnología como herramienta.

López-Torres, Maldonado, Pinzón y García (2016), escriben también sobre las actividades de innovación de las pymes y como su falta de implementación podría generar un desaprovechamiento en los recursos disponibles. Para OsorioGallego, Londoño-Metaute y LópezZapata (2016), el uso de las Tics es importante para que las mipymes sean competitivas y generen rentabilidad, porque permiten efectividad en las comunicaciones, reducción de costos, incentivos del gobierno. Además los autores se enfocan en entender porque las mipymes colombianas no adoptan estas tecnologías para su mejoramiento, determinando las barreras que tienen las empresas al no implementarlas.

\section{- Acceso a crédito}

Por medio de la revisión de literatura sobre los aspectos financieros que afectan a las Pymes de Latinoamérica, se determina que el acceso al crédito en países de la región es limitado, en parte por los requisitos y tasas de préstamo, por lo cual se quiere establecer qué factores influyen a que esta situación se presente.
Para Lecuona (2014), las empresas en América Latina presentan una restricción en el costo y acceso a los créditos, porque se financian con recursos internos y esto suele provocar problemas de solvencia en las empresas. También, Dong y Men (2014) reconocen los obstáculos y restricciones financieras en los países en desarrollo, puesto que dependen en gran medida de la financiación, disponibilidad de información crediticia, relación de concentración bancaria, desarrollo económico y entorno institucional.

Los autores Arráiz, Meléndez y Stucchi (2014) exponen sobre las garantías de créditos y rendimientos de las pymes, en donde es una problemática que las empresas no cuentan con solvencia propia, por lo que deben acceder a créditos y no cuentan con soportes suficientes para que se les sean otorgados. Los autores Vera-Colina, Melgarejo-Molina y Mora-Riapira (2014) abarcan el tema de acceso a financiación de estas empresas en Colombia, evidenciando que presentan indicadores deficientes de desempeño, lo que desencadena problemas con respecto a su capacidad de acceder a créditos por deficiente capacidad de endeudamiento.

De acuerdo con Saavedra y León (2014), el problema de la insolvencia radica desde el inicio de la empresa, cuando esta no cuenta con todos recursos necesarios para su funcionamiento óptimo, lo cual se ve reflejado en los indicadores de rentabilidad, liquidez y generación de valor, que la empresa no está desarrollando de manera correcta, por lo cual no puede cumplir con los requisitos del sistema financiero y se generan tasas de interés altas que cubran el riesgo de los préstamos.

Por su parte, Lopera, Vélez y Ocampo (2014) exponen que entre los errores que 
afectan a las mipymes se encuentra las pocas fuentes de financiación y el desconocimiento de otras fuentes, ocasionando restricciones de acceso al financiamiento generadas por problemas de asimetrías de información presentes en los mercados financieros. Por otro lado, Dini, Rovira y Stumpo (2014), analizan que para el crecimiento de las pymes es necesario la innovación, sin embargo, el apoyo financiero es limitado para el largo plazo y los programas no alcanzan los montos necesarios acordes para estos.

Según Rodríguez (2015), es importante analizar las limitaciones financieras que enfrentan las Pymes en Latinoamérica, donde se observa un acceso limitado al financiamiento, especialmente en el largo plazo. Para Botello (2015), el principal problema que las pymes colombianas presentan son las dificultades en sus indicadores, debido a la falta de acceso al crédito y la Fundación Observatorio Pyme (FOP, 2016a) establece el mismo problema en Argentina, debido a que genera estancamiento en el crecimiento, y la quiebra de las empresas.

\section{- Productividad y competitividad}

En esta sección, la problemática asociada a la productividad y competitividad radica en que las compañías pueden presentar riesgos, debido a que es un punto primordial para el mantenimiento de las pymes, pues la productividad depende la actividad de la empresa y la permanencia en el mercado depende de la competitividad.

Los autores Mayorga y Porras (2015), se enfocan en las pymes del sector de caucho y plástico analizando la problemática en la productividad de estas compañías, principalmente por aspectos tecnológicos, de capacitación y costos de producción, traducidos en problemas de capacidad que generan bajos márgenes de rentabilidad, los cuales afectan su funcionamiento. En el caso de Trujillo (2016), quien realiza un estudio a las pymes del sector de construcción en Colombia, donde concluye que tienen una baja productividad y rentabilidad, afectada por la carga financiera, entendiéndose esta como los costos que asume la empresa para su financiación. Además cuentan con una inadecuada gestión de los activos de operación, debido a que los administradores no manejan correctamentesus recursos o activos por desequilibrios en la rotación de activos, pasivos y capital de trabajo.

Igualmente, Leiva, Rodríguez y Monge (2017) en su artículo sobre la contratación de los ex-empleados de compañías multinacionales en empresas locales, expresan que los efectos de esta actividad en las pymes pueden mejorar el índice de capacidad de absorción de la empresa, debido a que existe un bajo desempeño y productividad por falta de capacidad y apoyo tecnológico. Los autores, Jara, et al (2014), mencionados anteriormente, determinan que las falencias en elementos de capacitación e inseguridad afectan la competitividad de los restaurantes.

Vanegas, Botero y Restrepo (2014), utilizan el modelo de lógica difusa para evaluar la competitividad empresarial, analizando la volatilidad del tipo de cambio y los precios que afecta la rentabilidad de la empresa. Para analizar la competitividad, Pomar, Rangel y Franco (2014) se enfocan en los problemas que tienen las Pymes de AguascalientesMéxico en donde encuentra barreras que afectan a las empresas, como la falta de recursos financieros, deficiencia en la comercialización, mal desempeño de recursos humanos y una deficiente gestión financiera. 


\section{- Planificación y asignación de recursos}

La forma como una empresa maneja sus recursos es fundamental para la duración y buenos resultados de ella, debido a que una incorrecta planificación o asignación de recursos ocasiona desbalances en las finanzas o pérdidas, que frecuentemente tienen un alto impacto sobre las Pymes. Por lo cual, se hace necesario revisar cuales son los factores por los que estas empresas no le dan importancia a la administración y gestión de recursos.

En este caso, Mora-Riapira, VeraColina y Melgarejo-Molina (2015) se refieren a la planificación estratégica y niveles de competitividad, donde recomiendan el fortalecimiento en las capacidades de los empresarios, y apoyo financiero, los cuales han sido un problema frecuente para lograr un mejor uso de los recursos y aumentar su desempeño, alcanzando un crecimiento empresarial.

Para los autores De León, Flores, Hernández, Resendiz y Yáñez (2015), las pymes pueden presentar dificultades cuando las decisiones que se tomen en las empresas no sean las adecuadas para las necesidades de las mismas, ellos explican que para esto es necesario hacer las lecturas de los estados financieros de manera correcta. De igual forma, la Fundación Observatorio Pyme (FOP, 2015), encuentra importante que las empresas cuenten con una planificación adecuada, contando con previsiones financieras para el hacer frente a los cambios coyunturales.

Siguiendo con un estudio sobre las pymes familiares, Dolz, Iborra y Safon (2015), determinan que las empresas con administradores miembros de la familia pueden generar soluciones o problemas a las mismas, dependiendo de la planificación y la respuesta que ellos quieran dar al mercado, su nivel de estudio, experiencia, su papel para delegar y contar con un equipo directivo. A la vez, Murcia (2015) analiza los problemas financieros que enfrentan las mipymes causados por un sesgo cognitivo del administrador que en su mayoría toma decisiones bajo incertidumbre, por no contar con los instrumentos adecuados.

Ottogalli, Rovhein, Roark, Urrutia, Paravie y Corre (2016) plantean que el desarrollo y madurez de una empresa comienza con conocer la disponibilidad de los recursos y como estos son gestionados, presentando así una falla en las pymes. Para Maliso y Aguirre (2016), la planeación financiera es una herramienta fundamental para la toma de decisiones en una empresa, sin embargo, su uso es limitado en pequeñas y medianas empresas por poca capacitación y ausencia de habilidades técnicas en administración y finanzas.

Para Molina, López y Schmitt (2016), los principales problemas a los que enfrentan las mipymes, desde el punto de vista de un grupo de expertos investigadores, es la baja planificación financiera, alto nivel de endeudamiento, cartera vencida excesiva, continuos incrementos en el costo de la materia prima, bajo nivel de ventas y utilidades y falta de capital para invertir en capital de trabajo. A la vez, Castro, Varela, Valencia, Oviedo, Molano y Carrillo (2016) explican que es importante que las mipymes tengan un control administrativo y financiero que les permita mantenerse a pesar de las carencias y dificultades respecto a la falta de herramientas de información financiera, las cuales afectan la rentabilidad y productividad generando riesgos y amenazas que los lleven al fracaso empresarial. 
Rodríguez y Vargas-Hernández (2017), determina las causas por las cuales las pymes están generando ambientes laborales lo que se traduce en altos índices de rotación de personal, encontrando como principal falla que los administradores toman decisiones sin realizar planeaciones si no siguiendo sus presentimientos. Respecto al manejo de la planeación también es importante fijarse en el horizonte temporal como lo establecen Molina, Botero y Montoya (2017), quienes plantean que el mercado permite orientar su planeación en el largo plazo y en la generación de valor.

\section{- Configuración de la estructura de capital}

Con respecto a este factor, que se refiere a la forma como las compañías manejan sus recursos para financiarse, se establece que una mala distribución o uso de los mismos puede ocasionar problemas financieros importantes para las Pymes.

Con respecto a este tema, Melgarejo, Vera y Mora (2014) denotan las falencias en la estructura de capital de las pymes debido a su distinta configuración, los problemas principales que enfrentan son de solvencia, rentabilidad y endeudamiento, y sobre todo este último que puede restringir su desarrollo. Para Vargas (2014), los problemas que enfrentan las pymes cuando existen costos de agencia, se representan en los costos de supervisión y contratación. Para disminuir el impacto de éstos costos, se puede utilizar el apalancamiento financiero, como mecanismo de control.

Por otro lado, Franco y Urbano (2014), con su artículo sobre el dinamismo de las pymes en Colombia, describen que una inestable estructura de capital genera iliquidez y con esto atraso en pago de proveedores y obligaciones de corto plazo.
De igual manera, para Novoa y Acevedo (2015), el no contar con una estructura de capital óptima genera riesgos de liquidez por lo cual se enfocan en encontrar cual es el punto de equilibrio entre la financiación interna y la externa.

Pérez, Bertoni, Satter y Domingo (2015), estudian cómo las Pymes de la comisión nacional de valores de Argentina muestran un alto endeudamiento con diversificación de su financiamiento, determinado que este tipo de empresas se enfocan en buscar distintas alternativas que subsanen riesgo de iliquidez que se presenta. Por otro lado, Villegas, Hernández y Salazar (2016), también visualizan el problema de las pymes con respecto a la dependencia de estas de financiación con créditos del sector privado.

\section{- Manejo financiero}

De acuerdo con la literatura revisada, la manera en la cual se lleva a cabo el manejo financiero en las pymes, puede llegar a ocasionar desequilibrios en el área financiera de las mismas, debido a que esta incluye aspectos de contabilidad, controles en pagos y auditorías mal manejadas.

Toro y Palomo (2014), en su análisis sobre riesgos financieros aplicado a Manizales realizan un estudio a $100 \mathrm{em}$ presas, de las cuales en 87 de ellas se estableció que poseían riesgos en varios indicadores financieros. El indicador con mayor porcentaje de riesgo fue el endeudamiento con promedio de $87 \%$, lo cual quiere decir que las empresas presentan problemas de manejo financiero, ya que comprometieron más del patrimonio que tenían en ese periodo.

Mendoza y Quintanilla (2015), tienen en cuenta que las condiciones financieras son inestables, lo que afecta el manejo de información y toma de decisiones de las 
pymes. Adicionalmente, Miranda, González y Castaño (2015) identifican que las mipymes no cuentan con una base contable adecuada en sus operaciones, ya sea por no darle la importancia necesaria o no tienen una debida formación empresarial.

También, Salas y Becerra (2015) identificaron algunos riesgos de quiebra de las pequeñas y medianas empresas por medio de los indicadores financieros, siendo algunos de ellos los bajos niveles de rentabilidad y liquidez. Para Asdraldo (2015), los problemas de las pymes como el poco apoyo de las entidades financieras y la debilidad contable que puede ser un riesgo para la continuidad de las pequeñas empresas, ya que el desconocimiento de la realidad de la empresa puede llevar a una toma de decisiones incorrecta.

Los autores Romero, Melgarejo y Vera (2015), en su trabajo sobre el fracaso de pymes en Colombia, exponen que un factor importante puede ser que sus ventas no crecen lo suficiente para solventar algunos costos y gastos, por lo que la rentabilidad se puede ver afectada. En el caso de Rodríguez y Mantilla (2016), quienes analizan las restricciones de las pymes respecto a la financiación y la frontera de posibilidades, donde aclaran las dificultades en la gestión del riesgo, las pymes no disponen de estados financieros auditados que permitan una mejor imagen de la empresa y dificultan el otorgamiento de préstamos.

La Fundación Observatorio Pyme (FOP, 2016b), estudia dos sectores productivos en Argentina. El primero es el de manufacturas y el segundo es el de software y servicios informáticos, este estudio se desarrolla con unas condiciones de mercados compleja en el país y concluye que la gestión y manejo de las empresas presenta problemas financieros para estas.Finalmente, Santana (2017) se refiere a los determinantes para la sobrevivencia de microempresas en la ciudad de Bogotá, en donde concluye que uno de los obstáculos está relacionado con la rentabilidad operativa, más exactamente del año inicial donde según su estudio debe ser alta para que se determine una posibilidad mayor de supervivencia en el mercado de estas empresas.

\section{- Políticas públicas}

Las políticas públicas hacen referencia a la serie de reglamentaciones que establecen los gobiernos de los países en cuanto al acceso de empresas a ciertos servicios, impuestos o requisitos que se deben cumplir, de allí que Chumaceiro y Hernández (2016) expresan: "las políticas públicas subordinan intereses e impulsan estrategias definidas como prioritarias en un determinado contexto y área de acción” (p. 106), en el ámbito internacional, los países desarrollados han puesto de manifiesto estrategias y políticas que incentivan a las mipymes ya que aumenta el potencial para contribuir al crecimiento sostenible del país (Chumaceiro, 2013). Sin embargo, se puede determinar que las pymes en Colombia se ven afectadas, y tienen la necesidad de acceder a los mercados internacionales y no hay políticas públicas suficientemente fuertes para apoyarlas.

Cardoza, Fornes, Farber, González, Gutiérrez y Ruiz (2014) se refieren a las barreras que afectan las pymes latinoamericanas y revisan los riesgos que tienen las empresas para realizar una expansión, por lo cual estudian las políticas públicas y otros impulsores como el acceso a los recursos financieros públicos, acceso a los contratos públicos, marcos legales adversos e inconsistentes, la asistencia pública en información y conocimiento sobre los mercados. 
Adicionalmente, Vázquez, Núñez y Fernández (2014), identifican las serias limitaciones de las pymes Latinoamericanas, como la falta de recursos financieros y escasos apoyos gubernamentales, que impiden el rendimiento y dificulta la ejecución de la innovación. Asimismo, Reina y Callejas (2015) explican las fallas de las pymes, orientada a los factores internos y externos como el desconocimiento para acceder a un crédito o las fluctuaciones de tasa de interés, que puede llegar a ser altas.

Ferrer, González y Mendoza (2015) identifican como el marco regulatorio afecta la gestión financiera en las pymes, las instituciones a cargo del diseño y la aplicación de las políticas, generando restricciones en materia de recursos humanos y financieros. En el caso de Vargas y Ovalle (2015), quienes realizan un estudio a las mipymes de la ciudad de Armenia, donde encuentran la existencia de un gran desconocimiento de la norma NTC 8201, lo que conlleva a una carente la relación entre la norma y los procesos que se están desempeñando.

Para el caso de Argentina, Briozzo, Vigier, Castillo, Pesce y Speroni (2016), determinan que existe una diferencia legal en la asignación de créditos a las empresas dependiendo de su tamaño, por lo cual se hace necesario la implementación de políticas públicas enfocadas al segmento de las pymes. Molina y Sánchez (2015) identifican el gran potencial que las Pymes tienen para generar competitividad, sin embargo, el mercado financiero latinoamericano no ofrece gran cantidad de alternativas de financiamiento por falta de políticas que permitan fomentar y proteger a las pymes, puesto que tienen una gran importancia económica y social.

En este orden de ideas, Briceño (2016) explica el poco apoyo por medio de polí- ticas públicas, lo que ocasiona que las empresas no sean productivas, bajos recursos destinados para I+D y dificultades para acceder a los instrumentos financieros. Se observa que los programas de innovación son diseñados de manera general y resultan de difícil acceso para pequeñas empresas.

Galindo y Micco (2016), determinan que una de las causas por las cuales las pymes tienen tasas de interés más altas es por la asimetría de información y la protección jurídica del sistema financiero. Igualmente, la Fundación Observatorio Pyme (FOP, 2016c), establece como en Argentina las dificultades del acceso al crédito se deben a los problemas estructurales de las políticas públicas que favorecen a la banca y se olvidan que las pymes tienen necesidades de financiamiento a tasas de interés competitivas.

Zuleta (2016) expone la falta de educación financiera de las pymes respecto a los créditos, siendo una falencia que les impide acceder al crédito por los niveles de tasas de interés y la complejidad de la documentación requerida. Asimismo, para Blanco, Chalá y Mercado (2016), las consecuencias de la inflación en las pymes, afecta la limitación para obtener créditos con una entidad financiera porque a menudo los intereses son altos a causa de la decisión que toma el Banco de la República para reducir la inflación.

Para analizar el efecto que la banca pública tiene en el escaso financiamiento de las pymes, Villar, Briozzo, Pesce y Fernández (2016) realizan un estudio comparado de Argentina y Brasil en donde encuentran que el acceso a los créditos por parte de las pymes está limitado por la falta de información, el problema de razonamiento de créditos y la segmentación de mercados financieros que se traduce en escenarios en donde falla el mercado. 
TABLA 1

Influencia de los factores identificados

\begin{tabular}{|c|c|c|c|}
\hline & $\begin{array}{l}\text { Factores que } \\
\text { originan las } \\
\text { problemáticas }\end{array}$ & Autores & Conclusión \\
\hline $\begin{array}{l}\text { Objetivos } \\
\text { financieros }\end{array}$ & $\begin{array}{l}\text { Innovación y } \\
\text { tecnología } \\
\text { Planificación y } \\
\text { asignación de } \\
\text { recursos } \\
\text { Productividad y } \\
\text { Competitividad } \\
\text { Manejo } \\
\text { financiero } \\
\text { Políticas } \\
\text { Publicas }\end{array}$ & $\begin{array}{l}\text { López-Torres, et al (2016) } \\
\text { Riascos, Aguilera y Achicanoy } \\
\text { (2016) } \\
\text { Cruz Pérez y Piedra (2014) y } \\
\text { García-Pérez, Gálvez-Albarracín y } \\
\text { Maldonado-Guzmán (2016). } \\
\text { Mora-Riapira, Vera-Colina y } \\
\text { Melgarejo-Molina (2015) } \\
\text { Ottogalli, Rovhein, Roark, Urrutia, } \\
\text { Paravie y Corre (2016) } \\
\text { León, Flores, Hernández, Reséndiz } \\
\text { y Yáñez (2015) } \\
\text { Fundación Observatorio Pyme } \\
\text { (FOP, 2015) } \\
\text { Molina, Botero y Montoya (2017) } \\
\text { Dolz, Iborra y Safon (2015) } \\
\text { Molina, López y Schmitt (2016) } \\
\text { Castro, Varela, Valencia, Oviedo, } \\
\text { Molano y Carrillo (2016) } \\
\text { Murcia (2015) } \\
\text { Maliso y Aguirre (2016) } \\
\text { Pomar, Rangel y Franco (2014) } \\
\text { Mendoza y Quintanilla (2015) } \\
\text { Miranda, González y Castaño } \\
\text { (2015) } \\
\text { Asdraldo (2015) } \\
\text { Rodríguez y Mantilla (2016) } \\
\text { Ferrer, González y Mendoza (2015) } \\
\text { Briceño (2016) } \\
\text { Vázquez Núñez y Fernández (2014) } \\
\text { Galindo y Micco (2016) } \\
\text { Fundación Observatorio Pyme } \\
\text { (FOP, 2016b) } \\
\text { Zuleta (2016) } \\
\text { Reina y Callejas (2015) } \\
\text { Blanco, Chalá y Mercado (2016) } \\
\text { Vargas y Ovalle (2015) } \\
\text { Villar, Briozzo, Pesce y Fernández } \\
\text { (2016) } \\
\text { Briozzo, Vigier, Castillo, Pesce y } \\
\text { Speroni (2016) }\end{array}$ & $\begin{array}{l}\text { La tecnología es un instrumento } \\
\text { de gestión debido a que permite el } \\
\text { acceso a la información completa } \\
\text { de la empresa, las Pymes no están } \\
\text { aprovechando de manera óptima las } \\
\text { oportunidades que el mercado les } \\
\text { ofrece. } \\
\text { La asignación de recursos y la } \\
\text { planificación son instrumentos clave } \\
\text { en una gestión administrativa. } \\
\text { Las Pymes no están estableciendo } \\
\text { horizontes temporales, ni } \\
\text { previniendo fallas del mercado y } \\
\text { sin esto no pueden cumplir con su } \\
\text { objetivo financiero. } \\
\text { Una deficiente gestión financiera } \\
\text { sin un enfoque claro a cumplir } \\
\text { los objetivos de la empresa, } \\
\text { no es competitiva y no maneja } \\
\text { adecuadamente los recursos y genera } \\
\text { fallas en la comercialización. } \\
\text { Si se realiza manejo financiero } \\
\text { inadecuado puede generar riesgos } \\
\text { que afecten la consecución de los } \\
\text { objetivos financieros. } \\
\text { Una política pública muy rígida } \\
\text { puede generar afectaciones en las } \\
\text { Pymes, puesto que a tasas de interés } \\
\text { muy altas las empresas deben pensar } \\
\text { en sacrificar ciertos recursos para } \\
\text { poder pagar los intereses. }\end{array}$ \\
\hline Liquidez & $\begin{array}{l}\text { Productividad y } \\
\text { Competitividad } \\
\text { Configuración } \\
\text { estructura de } \\
\text { capital } \\
\text { Manejo } \\
\text { Financiero }\end{array}$ & $\begin{array}{l}\text { Franco y Urbano (2014) } \\
\text { Novoa y Acevedo (2015) } \\
\text { Melgarejo, Vera y Mora (2014) } \\
\text { Pérez, Bertoni, Satter y Domingo } \\
\text { (2015) } \\
\text { Salas y Becerra (2015) }\end{array}$ & $\begin{array}{l}\text { Los problemas de liquidez en } \\
\text { las empresas, afectan de forma } \\
\text { directa la competitividad ya que se } \\
\text { atrasan procesos que son necesarios } \\
\text { diariamente, lo cual la pone en } \\
\text { desventaja en relación con las demás } \\
\text { empresas. } \\
\text { Las Pymes para disminuir los } \\
\text { riesgos de iliquidez deben determinar } \\
\text { un punto óptimo de su estructura de } \\
\text { capital. } \\
\text { La liquidez se ve afectada en como } \\
\text { las empresa gestionan sus riesgos, } \\
\text { manejan la incertidumbre, posibles } \\
\text { amenazas y problemas futuros. }\end{array}$ \\
\hline
\end{tabular}




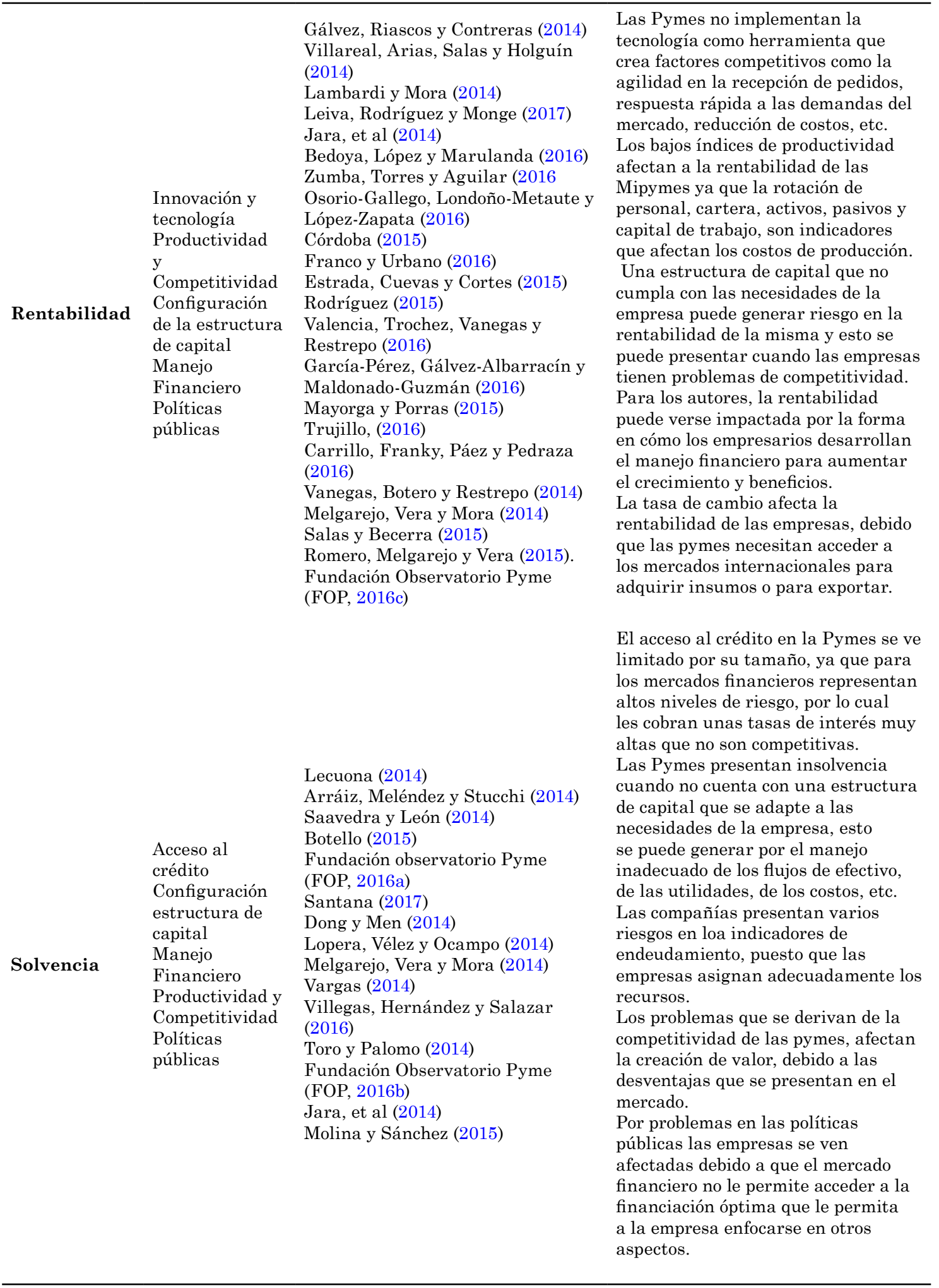

Fuente: Elaboración propia. 


\section{Metodología}

El enfoque de la investigación es cualitativo de alcance descriptivo y de tipo documental, ya que se basa en la revisión del estado del arte o literatura obtenida de bases de datos académicas como Ebsco, Jstor, Scielo, Science Direct y Scopus entre otras. Los criterios de búsqueda fueron las problemáticas financieras de las pequeñas y medianas empresas, teniendo en cuenta artículos posteriores al 2014 para generar actualizaciones en el tema propuesto. Posterior a la revisión de cada uno de los documentos, se procede a realizar una clasificación según el eje temático de cada artículo, obteniendo así los factores principales. Finalmente se establecieron las variables financieras que se ven afectadas por este conjunto de factores para así establecer un origen y consecuencias de las problemáticas en las pequeñas y medianas empresas y determinar las conclusiones extraídas de la revisión documental.

\section{Resultados}

Se identificaron los factores de innovación y tecnología, acceso a crédito, productividad y competitividad, planificación y asignación de recursos, configuración de la estructura de capital, manejo financiero y políticas públicas, como los que principalmente impactan a las pequeñas y medianas empresas en Bogotá, Colombia y América Latina según la revisión documental realizada. Adicionalmente se estableció como los objetivos financieros, la liquidez, solvencia y rentabilidad, generaban los principales impactos negativos en las empresas, los cuales originan las problemáticas financieras más comunes de las pymes.

\section{Conclusiones}

Como producto de la revisión del estado del arte realizado para establecer las problemáticas financieras que afectan a las pymes de Latinoamérica, se puede determinar que la manera como estas empresas se adminis- tran, afecta directamente la creación de valor, debido a las problemáticas encontradas, específicamente la baja productividad y competitividad, la falta de innovación, dificultad de acceso a créditos, políticas públicas ineficientes, inadecuada estructura de capital y manejo financiero incorrecto.

Entre los principales hallazgos de esta revisión de artículos, se encontró que la falta de tecnología e innovación llegan a afectar negativamente la generación de valor para las empresas y causan impactos negativos en aspectos financieros. Adicionalmente, una inversión en innovación y tecnología, en especial para los países en desarrollo resulta en aumentos de productividad para estas empresas. De igual manera, las tecnologías de información y comunicación son importantes porque las empresas que las implementen tienen mayor posibilidad de acceder a incentivos gubernamentales, reducir costos y manejar de manera adecuada sus recursos, ocasionando que las empresas sean más competitivas al reducir barreras.

Por otro lado, el acceso a la financiación de las pymes es limitado debido a que su operación es deficiente y presentan indicadores inadecuados para entidades bancarias, reflejando problemas de solvencia y capacidad de endeudamiento. Esto no les permite cumplir con los requisitos del sistema financiero, generando riesgo de crédito y como consecuencia tasas de interés elevadas. Igualmente, en el caso de las políticas públicas, se evidencia que existe poco apoyo gubernamental para las pymes, lo que dificulta que se le otorguen créditos a estas empresas, debido a factores como inflación, asimetría de la información e impuestos.

Con respecto a la productividad y competitividad, debido a la carga financiera y costos de la empresa, se disminuye su capacidad productiva y este factor se ve afectado por una gestión inadecuada de los activos y los recursos disponibles para la producción. Asimismo, un inadecuado manejo financiero puede afectar diferentes áreas de la compañía causado por falta de formación financiera de los administrativos, generando 
riesgos y pérdidas por decisiones erróneas en el manejo de recursos.

En la planificación y asignación de recursos, una incorrecta interpretación de estados financieros puede causar diagnósticos erróneos de las necesidades de las empresas. Adicionalmente, para las pymes latinoamericanas, se presenta otra problemática la cual es la ausencia de instrumentos y controles adecuados para la toma de decisiones, lo que no permite realizar una planeación financiera pertinente. Finalmente, una mala estructura de capital genera problemas de liquidez, afectando el cumplimiento de obligaciones de las empresas, y una inadecuada combinación de deuda y patrimonio puede aumentar los niveles de riesgo.

En la revisión bibliográfica, se observa que la mayor cantidad de factores que originan dichas problemáticas afectan principalmente a los objetivos financieros y la solvencia, lo que se ve reflejado en la destrucción de valor de las empresas, esto como resultado del manejo inadecuado de los demás factores como son la planificación y asignación de recursos, la innovación y tecnología, y el acceso al crédito y las dificultades de las políticas públicas. Teniendo en cuenta lo anterior, se evidencia que varios factores pueden llegar a impactar las variables financieras de las compañías, estableciendo retos en las empresas para controlarlos y sobre todo prevenirlos, estableciendo políticas de gestión adecuadas que favorezcan los objetivos de las empresas e impulsen su crecimiento y generación de valor.

\section{Recomendaciones}

Se considera importante realizar futuras investigaciones sobre el comportamiento de los objetivos financieros, la liquidez, rentabilidad y solvencia en las pequeñas y medianas empresas, para poder determinar por medio de estudios cuantitativos, las falencias y señales de la generación de problemas financieros, apoyando en correlaciones entre cuentas financieras o diferentes indicadores y profundizar en estas variables, obtenien- do conclusiones que aporten a la academia, economía y desempeño financiero de las empresas.

Se recomienda analizar la información presentada en esta revisión bibliográfica de manera cuantitativa, usando modelos econométricos para validar las relaciones encontradas y así desarrollar una guía para que las pymes encuentren información consolidada sobre los posibles problemas que puedan tener, diagnosticarlos en el momento adecuado para desarrollar medidas que los contrarresten de manera efectiva.

\section{Referencias}

Arráiz, I., Meléndez, M. \& Stucchi, R. (2014). Partial credit guarantees and firm performance: evidence from Colombia. Small Business Economics, 43(3). 711-724. https://doi.org/10.1007/s11187014-9558-4

Asdraldo, C. (2015). Prospectiva tecnológica como ventaja competitiva para la gestión financiera en las PYMES.Redes de ingeniería, 6(Edición especial). 113120. https://doi. org/10.14483/2248762X.8647

Bedoya, O., López, M. y Marulanda, C. (2017). Las buenas prácticas y las TI en la gestión del conocimiento de las Pymes Colombia. Revista espacios, 38(3). 7.

Blanco, S., Chalá, S. y Mercado, E. (2016). Inflación: un tema que afecta a las pymes de Medellín. Ágora Revista Virtual de Estudiantes. (4). 10-20. Recuperado de http://ojs.tdea.edu.co/index.php/ agora/article/view/372

Briceño, L. (2016). Desafíos de la política de innovación colombiana frente a las Pymes. (Tesis Magister). Universidad Militar Nueva Granada, Bogotá, D.C., Colombia.

Botello, H. (2015). Determinants of access to credit for SMEs: evidence at the level of the firm in latin America. Apuntes del CENES, 34(60). 247-276. 
Briozzo, A., Vigier, H., Castillo, N., Pesce, G. y Speroni, C. (2016). Decisiones de financiamiento en pymes: ¿existen diferencias en función del tamaño y la forma legal? Estudios Gerenciales, 32(138). 71-81. https://doi.org/10.1016/j.estger.2015.11.003

Carrillo, M., Franky, C., Páez, P. y Pedraza, A. (2016). Scloudpy: Sistema Informático Web de Multi-Tenencia para el Procesamiento en la Nube de Pedidos de PYMES. Información tecnológica, 27(1). 40-62. http://dx.doi.org/10.4067/ S0718-07642016000100019

Cardoza, G., Fornes, G., Farber, V., González, R. y Ruiz, J. (2016). Barriers and public policies affecting the international expansion of Latin American SMEs: Evidence from Brazil, Colombia, and Peru. Journal of Business Research, 69(6). 2030-2039.

Castro, F., Varela, J., Valencia, L., Oviedo, J., Molano, J. y Carrillo, J. (2016). Estado Administrativo contable y financiero de las tiendas de las comunas 5 de la ciudad de Ibagué. Vía Innova. 3(1). 1221.

Chumaceiro, A. (2013). Políticas internacionales, nacionales y políticas públicas tributarias en Venezuela como promotoras de la responsabilidad social de las empresas. (Tesis doctoral). Universidad Privada Dr. Rafael Belloso Chacín. Venezuela.

Chumaceiro, A. y Hernández, J. (2016). Países generadores de políticas públicas para el fomento de la cultura empresarial dirigida hacia la responsabilidad social. Revista Telos, 18(1). 103-120. Disponible en http://ojs.urbe.edu/index. php/telos/article/view/790.

Córdoba, M. (2015). Technology implementation as a strategy to enhance productivity and competitiveness of clothing manufacturing SMEs in Medellin. Trilogía, 7(12). 105-119.
Cruz, D., Pérez, S. y Piedra, V. (2014). Correlación de proyecciones financieras y situación financiera, con respecto a la competitividad e innovación en la PYME manufacturera de Cd. Sahagún, Hgo., México, para alcanzar su crecimiento empresarial. En, ASEPUC, Trasparencia y ética de la información financiera en las organizaciones públicas y privadas. Memorias XVI Encuentro ASEPUC. Murcia, España.

De León, I., Flores, I., Hernández, E., Reséndiz, C. y Yáñez, L. (2015). Análisis de la Administración Financiera desde la perspectiva de la interpretación de los estados financieros de las PyMes. XIKUA Boletín Científico de la Escuela Superior de Tlahuelilpan, 3(6). https:// doi.org/10.29057/xikua.v3i6.1311

Dini, M., Rovira, S. y Stumpo, G. (Comp.) (2014). Una promesa y un suspirar Políticas de innovación para pymes en América Latina. Santiago de Chile: Cepal.

Dolz, C., Iborra, M. y Safón, V. (2015). Empresa familiar, equipes administrativas, diversidade e ambidestriaem. $A d$ ministração de Empresas, 55(6). 673-687. http://dx.doi.org/10.1590/ S0034-759020150606

Dong, Y. y Men, C. (2014). SME financing in emerging markets: Firm characteristics, banking structure and institutions. Journal Emerging Markets Finance and Trade, 50(1). 120-149. https://doi.org/10.2753/REE1540496X500107

Estrada, S., Cuevas, H. y Cortés, H. (2015). El rendimiento de las MIPYMES industriales de Guanajuato a través de la innovación. En, D. Barbieux (Coord.) Innovación más allá de la tecnología, Anales del XVI Congreso Latino-Iberoamericano de Gestión Tecnológica ALTEC, Porto Alegre, Brazil. https://doi. org/10.13140/RG.2.1.3619.2402 
Ferrer, L., González, K. y Mendoza, L. (2015). La innovación como factor clave para mejorar la competitividad de las PYMES en el departamento del Atlántico, Colombia. Dictamen libre, 16(1).2136.

Franco, M. y Urbano, D. (2016). Factores determinantes del dinamismo de las pequeñas y medianas empresas en Colombia. Revista de Ciencias Sociales RCS, 22(1). 110-125.

Franco, M. y Urbano, D. (2014). The dynamism of SMEs in Colombia: A comparative study of companies. Revista Venezolana de Gerencia, 19(66). 319338.

FOP. (2016a). Informe Especial: Inversión y acceso al financiamiento en el segmento PyME manufacturero. Aspectos estructurales. Buenos Aires: FOP.

FOP. (2016b). La Coyuntura de las PyME industriales en el Tercer Trimestre 2016. Notas comparativas con la industria del Software y los Servicios Informáticos. Buenos Aires: FOP.

FOP. (2016c). Tipo de cambio y PyME industriales. Buenos Aires: FOP.

FOP. (2015). Industriales, informe Especial: El entorno local y el acceso a infraestructura de las PyME. Buenos Aires: FOP.

FOP. (2014). Informe Especial: El desempeno de las PyME industriales en 2014, con balance negativo. Buenos Aires: FOP.

Galindo, A. y Micco, A. (2016). Protección de los acreedores, intercambio de información y crédito para pequeñas y medianas empresas: datos comparativos. Revista de la CEPAL (120). 7-31.

Gálvez, E., Riascos, S. y Contreras, F. (2014). Influencia de las tecnologías de la información y comunicación en el rendimiento de las micro, pequeñas y medianas empresas colombianas. Estudios Gerenciales, 30(133). 355-364. https:// doi.org/10.1016/j.estger.2014.06.006
García-Pérez, D., Gálvez-Albarracín, E. y Maldonado-Guzmán, G. (2016). Efecto de la innovación en el crecimiento y el desempeño de las Mipymes de la Alianza del Pacífico. Un estudio empírico. Estudios Gerenciales, 32(141). 326-335. https://doi.org/10.1016/j.estger.2016.07.003

Jara, M., Guerrero, J., Jiménez, J., Hernández, E. y Rubio, J. (2015). Factores de competitividad de las pymes del sector de restaurantes en Bogotá, localidad la Candelaria. Revista Intersección, 1(1). 15-23.

Lambardi, G. y Mora, J. (2014). Determinantes de la innovación en productos o procesos: en el caso colombiano. Revista de Economía Institucional, 16(31). 251262.

Lecuona, R. (2014). Algunas lecciones de la experiencia reciente de financiamiento a las pymes. Santiago de Chile: Cepal.

Leiva, J., Rodríguez, J. y Monge, R. (2017). Efectos de la contratación de exempleados de multinacionales en la capacidad de absorción en empresas locales. Contaduría y Administración, 62(2). 670682. http://dx.doi.org/10.1016/j. cya.2016.06.009

Lopera, K., Vélez, V. y Ocampo, L. (2014). Alternativas de financiación para las Mipymes en Colombia. Revista virtual de los estudiantes de contaduría pública. Recuperado de https://aprendeenlinea.udea.edu.co/revistas/index.php/ adversia/article/download/20900/17492

López-Torres, G., Maldonado, G., Pinzón, S. y García, R. (2016). Colaboración y actividades de innovación en Pymes. Contaduría y Administración, 61(3). 568-581. https://doi.org/10.1016/j.cya.2015.05.016

Maliso, A. y Aguirre, M. (2016). Estudio de revisión sobre la planeación financiera y propuesta de modelo empírico para pymes de México. Revista CIMEXUS, 11(2), 73-106. https://dialnet.unirioja.es/ descarga/articulo/5886615.pdf 
Mayorga,J.yPorras,J.(2015).Productividad de las Pymes, sector caucho y plástico de Bogotá. D.C. Económicas CUC, 36(2). 95-111.

Melgarejo, Z., Vera, M. y Mora, E. (2014). Diferencias de desempeño empresarial de pequeñas y medianas empresas clasificadas según la estructura de la propiedad del capital, caso colombiano. Suma Negocios, 5(12). 76-84. https:// doi.org/10.1016/S2215-910X(14)70029-1

Mendoza, J. y Quintanilla, J. (2015). Papel de la Auditoría de Estados Financieros en las pymes. Revista Publicando, 277284.

Miranda, J. González, L. y Castaño, C. (2015). Diagnóstico de la gestión financiera integral en la Mipymes Navojoa, Sonora. En, ACACIA y UJED, XIX Congreso Internacional de Investigación en Ciencias. Durango, México.

Molina, D. y Sánchez, A. (2015). Factores de competitividad orientados a la pequeña y mediana empresa (PYME) en Latinoamérica: revisión de la literatura. Revista San Gregorio. (15). 104-111.

Molina, P., Botero, S. y Montoya, A. (2017). Estudios de rendimiento en las empresas de familia. Una nueva perspectiva. Estudios Gerenciales, 33(142). 76-86. https://doi.org/10.1016/j.estger. 2016.10.009

Molina, R., López, A. y Schimtt, C. (2016). Factores que influyen los problemas de emprendimiento de la Mipymes: una percepción de los expertos. Revista Internacional Administración y finanzas, 9(1). 95-113.

Mora-Riapira, E., Vera-Colina, M. y Melgarejo-Molina, Z. (2015). Planificación estratégica y niveles de competitividad de las Mipymes del sector comercio en Bogotá. Estudios Gerenciales, 31(134). 79-87. https://doi.org/10.1016/j.estger. 2014.08.001
Murcia, N. (2015). Los sesgos cognitivos en decisiones financieras que ocasionan la liquidación de Mipymes: un acercamiento a las Neurofinanzas. Ensayos: Revista de Estudiantes de Administración de Empresas, 8(8). 171-185.

Novoa, A. y Acevedo, E. (2015). Factores sectoriales clave para la estructura de capital en actividades de servicios petroleros en Colombia. Suma de Negocios, 6(14). 147-154.

ONU. Comisión Económica para América Latina y el Caribe-CEPAL. (s.f.). Micro, pequeñasy Medianas Empresas MiPyME. Disponible en https://www.cepal.org/es/ temas/micro-pequenas-medianasempresas-mipyme

Osorio-Gallego, C., Londoño-Mataute, J. y López-Zapata (2016). Análisis de los factores que influyen en la adopción de las TIC por parte de las PYMES de Colombia. Intangible Capital, 12(2). 698732. http://dx.doi.org/10.3926/ic. 726

Ottogalli, D., Rohvein, C., Roark, G., Urrutia, S., Paravié, D. y Corres, G. (2016). Desarrollo y madurez de las actividades de un conglomerado de PYMES industriales argentinas. Ingeniería Industrial, 15(1). $17-33$

Pérez, J., Bertoni, M., Sattler, S. y Domingo, D. (2015). Bases para un modelo de estructura de financiamiento en las pyme latinoamericanas. Cuadernos de Contabilidad. 16(40). 179-204. https://doi. org/10.11144/Javeriana.cc16-40.bpme

Pomar, S., Rangel, J. y Franco, R. (2014). La influencia de las barreras a la innovación que limitan la competitividad y el crecimiento de las Pymes manufactureras. Administracion y Organizaciones, 17(33), 33-57.

Reina, D. y Callejas, M. (2015). Causas de la siniestralidad de las MiPymes en Colombia: factores internos. (Master's thesis). Universidad EAFIT, Bogotá, D.C. Recuperado de https://repository.eafit. edu.co/handle/10784/7793 
Riascos, S., Aguilera, A. y Achicanoy, H. (2016). Inversión en tecnologías de la información y las comunicaciones y su relación con en el direccionamiento estratégico de las PYMES de Santiago de Cali - Colombia. RISTI - Revista Ibérica de Sistemas e Tecnologias de Informacao, (18). 1-17. http://dx.doi. org/10.17013/risti.18.1-17

Rodríguez, P. y Mantilla, D. (2016). Restricciones financieras de las pequeñas y mediana empresas. Revista Publicando, 3(9). 279-493.

Rodríguez, Á. y Vargas-Hernández, J. (2017). Plan de intervención organizacional para una Pyme de automatización: caso de Estudio Gisitca. Gestión Joven, 16(). 22-34.

Rodriguez, M. (2015). Indicadores del entorno de la pequeña y mediana empresa (PYME) en Latinoamérica, limitaciones a las que se enfrentan y fomento al desarrollo de las mismas caso Colombia. Revista Científica Guarracuco, 14(25). 57-82.

Romero, F., Melgarejo, Z. y Vera, M. (2015). Fracaso empresarial de las pequeñas y medianas empresas (pymes) en Colombia. Suma Negocios, 6(13). 29-41.

Saavedra, M. y León, E. (2014). Alternativas de financiamiento para la MIPyME. En, XIV Asamblea General de Alafec. Panamá.

Salas, G. y Becerra, M. (2015). Identificación de los factores de quiebra en las pymes de la localidad de Puente Aranda de Bogotá, Distrito Capital. Teoría y praxis investigativa, 9(2). 50-63.

Santana, L. (2017). Determinantes de la supervivencia de microempresas en Bogotá: un análisis con modelos de duración. Revista innovar journal, 27(64). 51-62. https://doi.org/10.15446/innovar.v27n64.62368
Toro, J. y Palomo, R. (2014). Análisis del riesgo financiero en las Pymes. Estudio de caso aplicado a la ciudad de Manizales. Revista Lasallista de Investigación, 11(2). 78-88. http://dx.doi. org/10.22507/rli.v11n2a9

Trujillo, A. (2016). Determinación de los factores financieros que influyen en el éxito y generación de valor de las pymes constructoras en Colombia. (Tesis Maestría). Universidad Nacional de Colombia, Medellín.

Valencia, M., Tróchez, J., Vanegas, J. y Restrepo, J. (2016). Modelo para el análisis de la quiebra financiera en pymes agroindustriales antioqueñas. Apuntes del CENES, 147-168.

Vanegas, G., Botero, C. y Restrepo, J. (2014). Una aproximación mediante lógica difusa al análisis de la competitividad empresarial. Administración y Organizaciones, 17(33). 9-32.

Vargas, L. y Ovalle, A. (2015). Pymes manufactureras de la ciudad de Armenia y sus procesos de innovación. Contexto, 4(1). 21-32.

Vargas, A. (2014). Estructura de capital óptima en presencia de costos de dificultades financieras. Investigación y desarrollo, 1(14). 44-65.

Vázquez, G., Núñez, T. y Fernández, J. (2014). Impacto de las operaciones en la competitividad en las pymes manufactureras en la zona metropolitana de Guadalajara. Revista de Investigación y Análisis, 15(1). 73-91.

Vera-Colina, M., Melgarejo-Molina, Z., y Mora-Riapira, E. (2014). Acceso a la financiación en Pymes colombianas: una mirada desde sus indicadores financieros. Revista Innovar Journal, 24(53). 149-160. https://doi. org/10.15446/innovar.v24n53.43922 
Villar, L., Briozzo, A., Pesce, G. y Fernández, A. (2016). El rol de la banca pública en el financiamiento Estudio comparativo para la Argentina. Desarrollo y Sociedad, (76). 205-241. http://dx.doi. org/10.13043/DYS.76.5

Villareal, N., Arias, D., Salas, N. y Holguín, H. (2014). Determinantes de la innovación y la productividad en la industria manufacturera colombiana por tamaño de firma. Observatorio Colombiano de Ciencia y Tecnología y el Departamento Nacional de Planeación, 1-48.

Villegas, E., Hernández, M. y Salazar, B. (2017). La medición del capital intelectual y su impacto en el rendimiento financiero en empresas del sector industrial en México. Contaduría y Administración, 62,(1). 184-206. http:// dx.doi.org/10.1016/j.cya.2016.10.002

Zuleta, A. (2016). Inclusión financiera de la pequeña y mediana empresa en Colombia 2016. Santiago: Naciones Unidas.

Zumba, M., Torres, G. y Aguilar, B. (2016). Los medios sociales: una nueva herramienta para la gestión de la innovación en las Pymes. Análisis en el Sector Servicios de la Región Sur del Ecuador. 11 Iberian Conference on Information Systems and Technologies. Recuperado de http://dspace.utpl.edu.ec/handle/123456789/18756
Ana María Gamez Tellez es Profesional en Finanzas y Comercio Internacional egresada del programa de finanzas y comercio internacional de la Universidad de La Salle: Bogotá, D.C. (Colombia)

Mabel Cristina Morales Bautista es Profesional en Finanzas y Comercio Internacional egresada del programa de finanzas y comercio internacional de la Universidad de La Salle: Bogotá, D.C. (Colombia)

Cindy Tatiana Ramirez Lopez es Profesional en Finanzas y Comercio Internacional egresada del programa de finanzas y comercio internacional de la Universidad de La Salle: Bogotá, D.C. (Colombia) 